\title{
Terminal Condylar Facets of the Femur: Implications for Knee Arthroplasty
}

\author{
Sunita Athavale, MD ${ }^{1}$ Sheetal Kotgirwar, MD, DNB ${ }^{1}$ \\ Soumitra Trivedi, MS ${ }^{4}$ Rekha Lalwani, MD ${ }^{1}$ \\ ${ }^{1}$ Department of Anatomy, All India Institute of Medical Sciences, \\ Bhopal, Madhya Pradesh, India \\ 2 Department of Anatomy, People's College of Medical Sciences and \\ Research Center, Bhopal, Madhya Pradesh, India \\ ${ }^{3}$ Department of Anatomy, Andaman \& Nicobar Islands Institute of \\ Medical Sciences, Port Blair, Andaman and Nicobar, India \\ ${ }^{4}$ Department of Anatomy, All India Institute of Medical Sciences, \\ Raipur, Chhattisgarh, India
}

J Knee Surg 2018;31:433-438.

\author{
Address for correspondence Sunita Athavale, MD, Department of \\ Anatomy, All India Institute of Medical Sciences, C-166 Emerald Park \\ City, Bhopal, Madhya Pradesh, India \\ (e-mail: sunita.anatomy@aiimsbhopal.edu.in).
}

\begin{abstract}
Keywords

- condyles

- high-flexion knee arthroplasty

- posterior horn

- total knee arthroplasty

In wake of changing lifestyle and increasing surgical expertise, knee replacement surgeries have become common. Attempts at improving the postoperative range of motion have prompted high-flexion knee arthroplasties. Consequently, there is a need to study the anatomy of knee joint in high flexion. This study focused on a holistic description of terminal condylar articular facet (TCAF; part of the femoral articular area in contact with the tibia in high flexion) of medial and lateral condyles and their quantification. In total, 104 dry femora and 16 dissected cadaveric knee joints (10 adult and 6 fetal) were studied. In most cases, the medial TCAF showed a tongue- or wedge-shaped extension in its lateral half, which has been referred to as extended condylar facet (ECF). The surface area of the ECF was approximately $0.5 \mathrm{~cm}^{2}$. In adult cadaveric dissections, the fibrous capsule showed the lax attachment beyond the ECF, thus creating a cave in the medial tibiofemoral compartment. These features were absent in fetal dissection. The presence of an ECF has not been documented in dry bones and has not been quantified. The variability of the size of this extension supports the hypothesis of this being an acquired trait. This study is expected to provide factual data for implant design improvement and a new anthropological data on Indian femora.
\end{abstract}

Total knee replacement surgeries are on the rise, probably due to changing lifestyles and also increased surgical expertise. Postreplacement joint movements are constrained in flexion. ${ }^{1}$ The range of motion of knee achieved postsurgery is considered to be one of the most important factors that determine the success of the surgery. ${ }^{2}$

During past few years, attempts to improve knee flexion have been made, and modified knee prostheses have been designed. These prostheses have aimed at component design modifications, which focused on the following:

- Lengthening the radius of curvature through the posterior condyles.

- Increasing the posterior condylar offset. ${ }^{3}$

received

July 28, 2016

accepted after revision

May 31, 2017

published online

July 18, 2017
However, systematic reviews on high-flexion knee arthroplasties suggest that there is not much improvement in range of motion achieved postoperatively. ${ }^{4-8}$

There are only two published studies describing tibiofemoral joint in hyperflexion (120 and 160 degrees) despite the relevance of this arc to total knee replacement. ${ }^{9,10}$ Nakagawa et $\mathrm{al}^{9}$ have suggested that at full flexion (162 degrees), the lateral femoral condyle is posteriorly subluxated, but the medial condyle still lies over the tibia but lifts away from it. Pinskerova et $\mathrm{al}{ }^{10}$ observed that in full flexion, the medial femoral condyle rises up on the posterior horn of the medial meniscus.

While these two studies reported that there are differences in sagittal profiles of femoral condyles on the medial and lateral sides, they were conducted on a small number of

Copyright $\odot 2018$ by Thieme Medical Publishers, Inc., 333 Seventh Avenue, New York, NY 10001, USA.

DOI https://doi.org/ 10.1055/s-0037-1604140. ISSN 1538-8506. 
specimens, and their findings were mostly based on sectional images and hence have stopped short of holistic description and quantification of the differences.

This study addressed the following questions:

- Are there any differences in condylar morphology of terminal ends of condylar facets on medial and lateral condyles of the femur? If yes, in what measure?

- Are there any differences in joint morphology of medial and lateral tibiofemoral compartments of the knee joint in adults and in fetuses?

The findings of this study can have bearing on designing of femoral component of knee prosthesis for high-flexion knee arthroplasty.

\section{Materials and Methods}

The cadavers and the dry femora required for the study were obtained from the collection of cadaveric material and the dry bones available in the Department of Anatomy (People's College of Medical Sciences and Research, Andaman \& Nicobar Islands Institute of Medical Sciences, Port Blair, India). Fully ossified dry femora and knee joints of adult cadavers were included. Femora with any congenital/acquired deformity, and bones showing any evidence of osteoarthritis at the lower end of femur were excluded. Five right and five left knee joints were used for the study. Age of cadaveric limbs was not known as cadaveric material was mostly obtained from unclaimed dead bodies without any proof of age or any other particulars. Sex of the limbs could not be ascertained as the limbs used were from the collection of limbs disarticulated at the hip joint from the torso, with no available documentation of sex.

Cadavers with any congenital/acquired deformity of the lower limb, and knee joints showing any evidence of osteoarthritis were also excluded.

The fetuses included in the study were from the collection of the department of anatomy with a gestational age of 16 to 36 weeks. Fetal specimens were included to observe the differences between the medial and lateral tibiofemoral joint compartments in utero.

\section{Bony Study}

In total, 104 femora (56 right; 48 left) were included in the study. The study intended to observe the terminal ends of femoral condylar facets on both the condyles. The study intended to observe the terminal ends of medial and lateral femoral condylar facets. The terminal ends were defined as part of the condylar articular area lying anterior to an imaginary horizontal line (L1). To mark L1, the outer articular margins of condyles were followed anteriorly toward the shaft and L1 was drawn across the point (P). Point $P$ was defined as a point on the outer margins of the condylar articular area (either medial or lateral) that starts curving inward. The area thus demarcated was called terminal condylar articular facet (TCAF). The observations were recorded as follows (-Fig. 1):

- The femur was fixed onto the osteometric board in anatomical position. A slider attachment (slider I) was

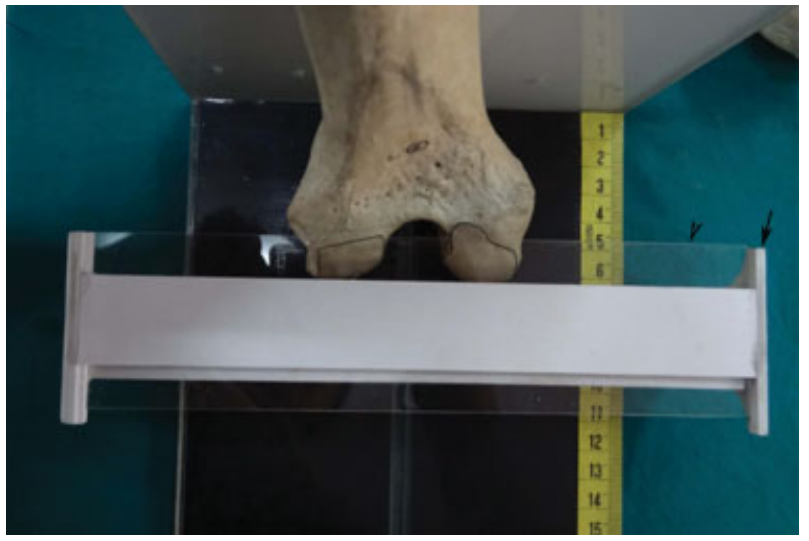

Fig. 1 The apparatus used and the method of demarcating the terminal condylar articular facet and extended condylar facet on dry femora. Arrow, slider 1; arrowhead, slider 2.

designed which could move on the osteometric board forward and backward.

- This slider, on its top, had another slider attachment (slider II) in the form of a transparent rectangular strip.

- After fixing the femur on the osteometric board, slider I was slid onto the board till it touched the posterior surfaces of the condyles. Slider II was then moved forward while inspecting the outer margins of articular areas through the transparent strip.

- After reaching point P as defined earlier, a horizontal line L1 was drawn on the femoral condyles using the anterior face of transparent strip as scale. This was intended to demarcate the TCAF in otherwise curved femoral condyles.

- Slider II was further moved anteriorly till the termination of the lateral condylar articular area was reached. Another horizontal line (L2) was drawn at this level, on the medial condyle, which represented the demarcation of extension of the articular area of the medial condyle beyond the lateral, henceforth referred to as extended condylar facet (ECF).

- The TCAF and ECF thus demarcated were then marked with a pencil and were traced on to a translucent butter paper. The surface area of medial and lateral TCAF and ECF were calculated using a digital planimeter. The measurements were analyzed statistically for the differences between lateral and medial condyles and right and left sides.

- - Fig. 2 shows the TCAF on the medial and lateral condyles, and ECF on the medial condyle as demarcated by the procedure described previously.

Each component of the bony study such as demarcation of the TCAF and ECF; translation of these on translucent paper; and measurement by planimetry was performed by individual observers to avoid interobserver bias. For morphometric component, observations were recorded thrice by the same observer and mean was taken.

\section{Cadaveric Study}

Sixteen cadaveric knee joints were also dissected (10 adult [ 5 right and 5 left] and 6 fetal [ 3 right and 3 left]). The muscles 


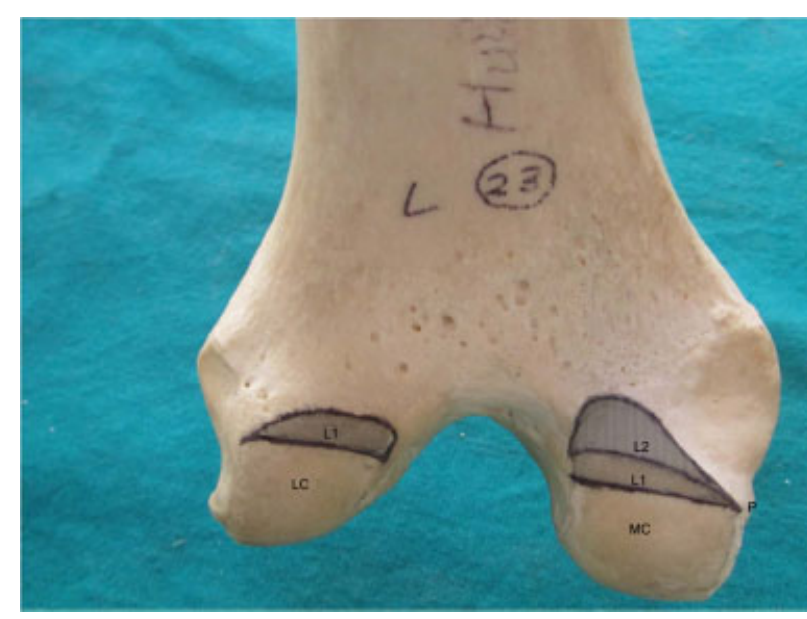

Fig. 2 Showing the terminal condylar articular facet (shaded gray) and extended condylar facet (patterned) demarcated by $L 1$ and $L 2$ on the femur. $\mathrm{P}=$ point across which $\mathrm{L} 1$ has been drawn. LC, lateral condyle; MC, medial condyle.

and ligaments surrounding the knee joint were cleaned and retracted/cut. The fibrous capsule of the knee joint was cut through a horizontal incision at the level of joint cavity and reflected. The morphology of the posterior aspects of the medial and lateral joint compartments was observed.

Following observations were documented regarding the joint capsule around the TCAF; all observations were made by single observer:

- Attachment of fibrous capsule.

- Any gap in the capsule.

- Any outpouching of the synovial membrane.

\section{Data Analysis}

The observations were recorded in tabular form separately for right and left sides. Descriptive statistics was performed on statistical software SPSS 20 version. Tests of significance were applied to the surface areas obtained from lateral and medial condyles and also between the right side and the left side.

\section{Observations}

The naked eye observation of the femoral condyles showed apparent differences between the medial and lateral sides. The posterior articular margin of the medial TCAF is nonlinear and, when traced laterally, shows a tongue-shaped extension in its lateral half. The posterior margin of the lateral TCAF was linear with slight obliquity or more or less horizontal (-Fig. 3). - Table 1 shows the surface areas of medial and lateral TCAF and ECF on the right and left sides. The difference between the TCAF of the right and left sides was statistically not significant, but that between medial and lateral sides was statistically highly significant $(p \leq 0.001)$.

It was observed that in most cases (99 out of 104), the medial TCAF extended further anteriorly beyond the lateral. This extension (ECF) was either in the form of tongue shape or a wedge (edge of the wedge directed medially) (-Fig. 2). In five femora ( 3 right and 2 left), the ECF was absent, that is, the medial TCAF did not extend beyond the lateral. It was observed that the ECF measured approximately $0.5 \mathrm{~cm}^{2}$.

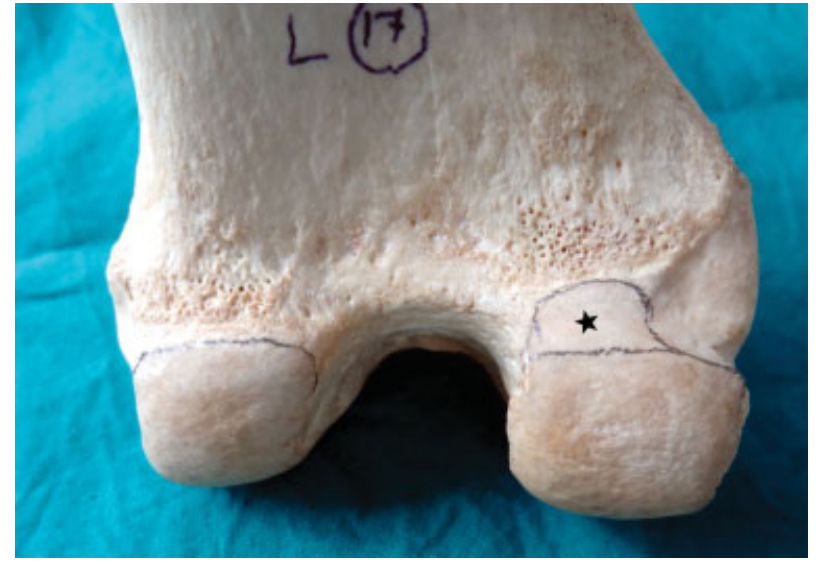

Fig. 3 Showing differences in posterior articular margins of medial and lateral condyles of the femur and tongue-shaped extension (denoted by ${ }^{*}$ ) of the medial terminal condylar articular facet. LC, lateral condyle; MC, medial condyle.

Cadaveric observations suggest that the attachment of the fibrous capsule around the TCAF on the lateral condyle was closely abutting the articular margin. On the medial condyle, however, fibrous capsule was closely abutting on the medial half of the TCAF but moved further away anteriorly from the articular margin including nonarticular part of bone covered by synovium, thus creating a cave/pouch in the medial tibiofemoral compartment (-Figs. 4 and 5). A constant feature observed in all cadaveric dissections was the presence of an oval-to-round gap of approximately half-a-centimeter diameter in the fibrous capsule in the lateral half of the medial tibiofemoral compartment. The capsular attachment on the lateral condyle was closely abutting the articular margin. A small incursion of the attachment of the fibrous capsule toward the joint cavity in the middle of the articular margin created two very shallow pouches on the lateral tibiofemoral compartment ( - Figs. 5 and $\mathbf{6}$ ).

The fetal cadaveric observation suggests that the articular area of the lateral condyle was horizontal and that of the medial condyle was oblique. But the medial condyle lacked the tongue-shaped extension ( $\mathbf{- F i g . ~ 7 ) . ~ T h e ~ c a v e / p o u c h ~ i n ~}$ the medial tibiofemoral compartment as observed in adults could not be discerned in fetal knee joints.

\section{Discussion}

Findings of this study (both bony and cadaveric) document the difference of joint morphology in the terminal ends on the medial and lateral tibiofemoral compartments in adults.

Standring et $\mathrm{al}^{11}$ describe that the sagittal profiles of femoral condyles are somewhat controversial. Provision of an extra articular space, and its shape and size on the medial side on the femoral condyle had not been documented earlier.

It is known that the medial tibial condylar articular area is more extensive anteroposteriorly as compared with the lateral one. On the medial condyle of the femur, the ECF, which is an extension of the TCAF on the medial femoral condyle, should suffice for articulation with tibial condyle in 


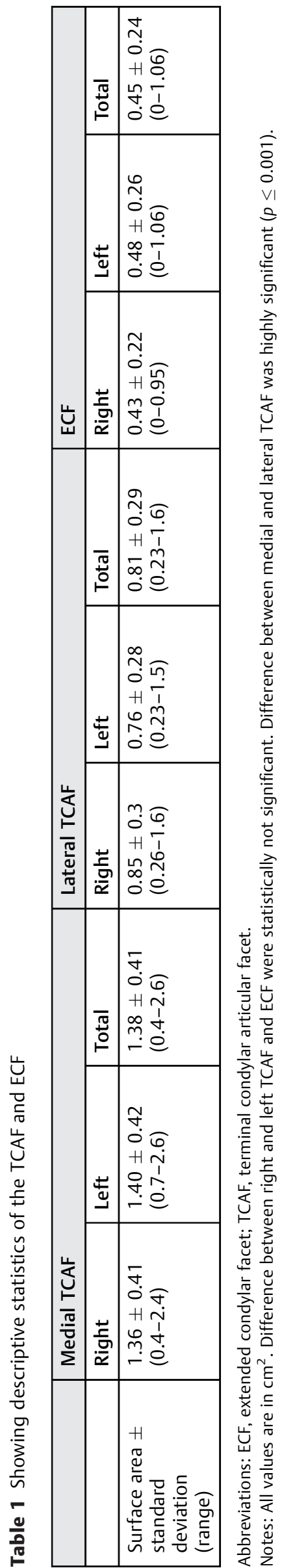



Fig. 4 Showing the difference in medial and lateral tibiofemoral compartments of knee joint. LC, lateral condyle; MC, medial condyle.

hyperflexion. In this study, the medial TCAF was found to be larger by approximately $0.5 \mathrm{~cm}^{2}$ than the lateral TCAF and extended beyond it.

The difference in morphology of the medial and lateral joint compartments in cadavers as observed in this study has been also documented by Pinskerova et al. ${ }^{10}$ Our findings of cadaveric specimen also confirm their findings, which they have called posterior horn space, with the following additions: (1) There is a constant gap in the fibrous capsule surrounding the ECF with outpouching of synovial membrane and (2) the fibrous capsule in the lateral compartment snuggly fits the articular margins with an incursion in the center, creating two shallow pouches.



Fig. 5 A sketch of knee joint showing medial and lateral tibiofemoral compartments, attachment of the fibrous capsule along the terminal condylar articular facets, and gap in the fibrous capsule (arrow). FC, fibrous capsule; LC, lateral condyle; MC, medial condyle. 




Fig. 6 Showing the gap in fibrous capsule (arrow). MC, medial condyle.

The differences between the lateral and medial tibiofemoral compartments underscore the functional importance of this area in high flexion of knee joint. It can be inferred that there is a provision of an extended articular area (ECF) on the medial femoral condyle for high flexion.

Many workers have emphasized the effect of posture on molding of articular areas, for example, tibia and talar articular facets of the talus, ${ }^{12,13}$ and tibial retroversion. ${ }^{14}$ Repeated assumption of hyperflexed position, as is common in Indians during squatting and palthi (leg-folding) posture in many day-to-day activities, might predispose to modeling of the TCAF and ECF. The variability of the size of this

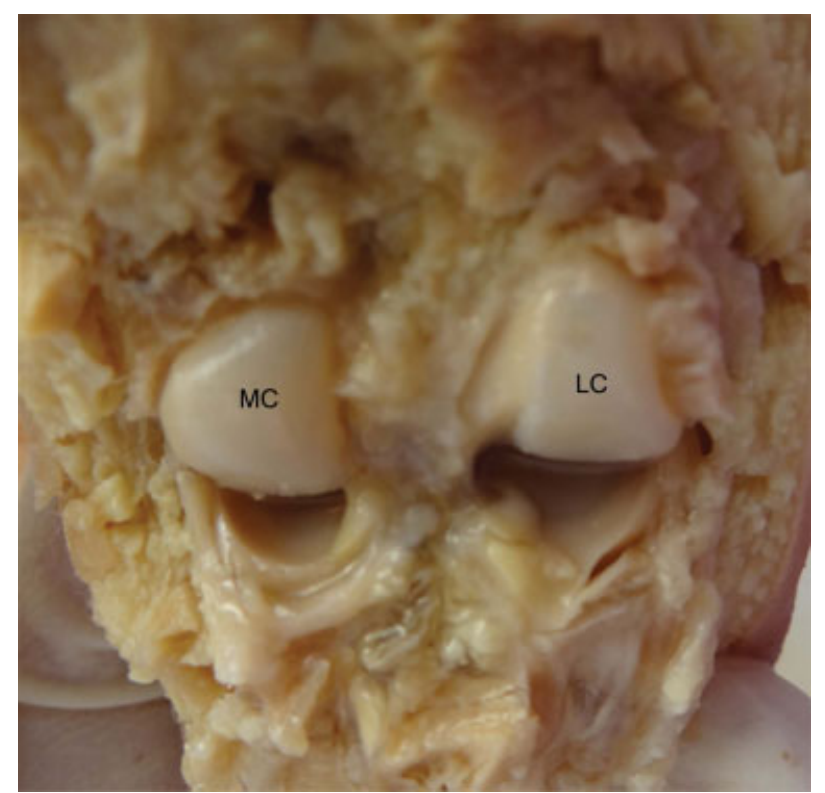

Fig. 7 Showing medial and lateral tibiofemoral compartments of fetal knee joint. LC, lateral condyle; MC, medial condyle. extension supports the hypothesis of this being an acquired trait. Squatting facets on the lower end of the medial condyle of the femur have also been described. ${ }^{15,16}$ However, the location and morphology of these facets described as "deep notch or smooth oval depression inferomedial to the adductor tublercle" resemble more closely with the description of adductor tubercle by Standring et al ${ }^{11}$ " as may only be a facet rather than projection" as described by and should not be confused with the ECF. As regards the occurrence of squatting facets on the upper end of the tibia is concerned, there is no description in the available literature. Authors feel that only the ECF is in articulation with the tibial condylar articular area in high flexion, and no other part of the femur is in contact with the tibia and therefore it is unlikely that any other facets or impressions would be observed in the tibia. The findings of the fetal dissections were only observational; however, the absence of any apparent extension of the articular area and laxity of the fibrous capsule on the medial side also indicate that the extension of the medial facet as observed in adults might be a manifestation of assumption of hyperflexed position frequently in day-to-day life. However, whether this is a congenital or acquired trait can be concluded only after further studies on different age groups and people with varying lifestyles.

This study is expected to provide factual data for implant design improvement. The ECF, if recreated in implant designs, will provide surface for accommodation of the medial condyle of the tibia with overlying posterior horn of medial meniscus with the femur in hyperflexion. This study also provides a new anthropological data on Indian femora, which is expected to promote comparative studies from other population groups.

The limitations of the study include the following. The study does not provide separate morphometric observation for male and females. Lifestyle, namely, squatting or nonsquatting behaviors, which can possibly influence the morphology of the TCAF, was not known. In the absence of concrete reliable landmark to delineate the TCAF, point P was defined and used. There can be slight error in recognizing point $P$. This was tided over by demarcating the TCAF by a single observer. Moreover, demarcation was done by a single horizontal line drawn across the two condyles with the help of sliding osteometric board as explained in the Material and Methods section earlier. Even in case of slight error in recognizing point $P$, the demarcation of, and thus the measurement of, two TCAFs (medial and lateral) would be affected equally. The cadaveric study was only observational, and no morphometric comparison between adult and fetal observations could be drawn owing to the very small size of fetal specimen.

\section{References}

1 Rowe PJ, Myles CM, Nutton R. The effect of total knee arthroplasty on joint movement during functional activities and joint range of motion with particular regard to higher flexion users. J Orthop Surg (Hong Kong) 2005;13(02):131-138

2 Murphy M, Journeaux S, Russell T. High-flexion total knee arthroplasty: a systematic review. Int Orthop 2009;33(04):887-893 
3 Long WJ, Scuderi GR. High-flexion total knee arthroplasty. J Arthroplasty 2008;23(7, Suppl):6-10

4 Hakki S, Saleh KJ, Potty AG, Bilotta V, Oliveira D. Columbus navigated TKA system: clinical and radiological results at a minimum of 5 years with survivorship analysis. Orthopedics 2013;36(03):e308-e318

5 McCalden RW, MacDonald SJ, Bourne RB, Marr JT. A randomized controlled trial comparing "high-flex" vs "standard" posterior cruciate substituting polyethylene tibial inserts in total knee arthroplasty. J Arthroplasty 2009;24(6, Suppl):33-38

6 Suggs JF, Kwon YM, Durbhakula SM, Hanson GR, Li G. In vivo flexion and kinematics of the knee after TKA: comparison of a conventional and a high flexion cruciate-retaining TKA design. Knee Surg Sports Traumatol Arthrosc 2009;17(02):150-156

7 Bollars P, Luyckx JP, Innocenti B, Labey L, Victor J, Bellemans J. Femoral component loosening in high-flexion total knee replacement: an in vitro comparison of high-flexion versus conventional designs. J Bone Joint Surg Br 2011;93(10):1355-1361

8 Namba RS, Inacio MC, Cafri G. Increased risk of revision for high flexion total knee replacement with thicker tibial liners. Bone Joint J 2014;96-B(02):217-223
9 Nakagawa S, Kadoya Y, Todo S, et al. Tibiofemoral movement 3: full flexion in the living knee studied by MRI. J Bone Joint Surg Br 2000;82(08):1199-1200

10 Pinskerova V, Samuelson KM, Stammers J, Maruthainar K, Sosna A, Freeman MA. The knee in full flexion: an anatomical study. J Bone Joint Surg Br 2009;91(06):830-834

11 Standring S, Ellis H, Healy JC, et al. Knee. In: Gray's Anatomy. New York, NY: Churchill Livingstone; 2005:1474-1477

12 Thomson A. The influence of posture on the form of the articular surfaces of the tibia and astragalus in the different races of man and the higher apes. J Anat Physiol 1889;23 (Pt 4):616-639

13 Singh I. Squatting facets on the talus and tibia in Indians. J Anat 1959;93:540-550

14 Boulle E-L. Evolution of two human skeletal markers of the squatting position: a diachronic study from antiquity to the modern age. Am J Phys Anthropol 2001;115(01):50-56

15 Kumar A, Koranne SP. The squatting facet on femora of West Coastal Indian population. Forensic Sci Int 1983;21(01):19-21

16 Kumar A, Shivarama C. The squatting facet on femora in South Indians. Int J Sci Res Educ 2015;3(07):3995-3997 